Published in final edited form as:

Mov Disord. 2009 July 30; 24(10): 1461-1467. doi:10.1002/mds.22571.

\title{
Validation of the Questionnaire for Impulsive-Compulsive Disorders in Parkinson's Disease (QUIP)
}

\author{
Daniel Weintraub, MD ${ }^{1,2,3,4}$, Staci Stewart, BA ${ }^{1}$, Judy A. Shea, $\mathrm{PhD}^{5,6}$, Kelly E. Lyons, \\ $\mathrm{PhD}^{7}$, Rajesh Pahwa, MD7, Erika D. Driver-Dunckley, $\mathrm{MD}^{8}$, Charles H. Adler, MD, $\mathrm{PhD}^{8}$, Marc \\ N. Potenza, MD, PhD $^{9}$, Janis Miyasaki, MD, MEd, FRCPC ${ }^{10}$, Andrew D. Siderowf, MD, \\ MSCE$^{2}$, John E. Duda, MD ${ }^{2,3}$, Howard I. Hurtig, M.D. ${ }^{2}$, Amy Colcher, MD ${ }^{2}$, Stacy S. Horn, \\ $\mathrm{DO}^{2}$, Matthew B. Stern, MD ${ }^{2,3}$, and Valerie Voon, MD ${ }^{11}$ \\ 1 Department of Psychiatry, University of Pennsylvania, Philadelphia, PA \\ 2 Department of Neurology, University of Pennsylvania, Philadelphia, PA \\ ${ }^{3}$ Parkinson's Disease Research, Education and Clinical Center (PADRECC), Philadelphia Veterans \\ Affairs Medical Center, Philadelphia, PA \\ ${ }^{4}$ Mental Illness Research, Education and Clinical Center (MIRECC), Philadelphia Veterans Affairs \\ Medical Center, Philadelphia, PA
}

\footnotetext{
Corresponding Author: Daniel Weintraub, MD, 3615 Chestnut St., Room 330, Philadelphia, PA 19104-2676, phone: (215) 349-8207, fax: (215) 349-8389, weintrau@ mail.med.upenn.edu.

Supplementary material: Four versions of the QUIP (full and short versions that assess for ICDs currently or anytime during PD) and scoring instructions are available online as supplementary material.

Authors' Contributions:

Daniel Weintraub, MD: study conception and execution; statistical analysis design, execution, and review and critique; and manuscript first draft preparation, review and critique

Staci Stewart, BA: study conception and execution; statistical analysis execution; and manuscript first draft preparation, review and critique

Judy A. Shea, PhD: study conception; statistical analysis design, review and critique; manuscript review and critique

Kelly E. Lyons, PhD: study execution and manuscript review and critique

Rajesh Pahwa, MD: study execution and manuscript review and critique

Erika D. Driver-Dunckley, MD: study execution and manuscript review and critique

Charles H. Adler, $M D$, PhD: study execution and manuscript review and critique

Marc N. Potenza, $M D, P h D$ : study conception; statistical analysis review and critique; and manuscript review and critique

Janis Miyasaki, MD: study conception and manuscript review and critique

Andrew D. Siderowf, $M D$ : study conception and execution; manuscript review and critique

John E. Duda, MD: study conception and execution; manuscript review and critique

Howard I. Hurtig, MD: study conception and execution; manuscript review and critique

Amy Colcher, $M D$ : study execution and manuscript review and critique

Stacy S. Horn, DO: study execution and manuscript review and critique

Matthew B. Stern, MD: study conception and execution; statistical analysis review and critique; and manuscript review and critique Valerie Voon, MD: study conception and manuscript review and critique

Financial Disclosures: Dr. Weintraub has received grant support from NIH and Boehringer Ingelheim, and consulting fees or honoraria from Boehringer Ingelheim, BrainCells, EMD Serono, Novartis, Ovation, and Wyeth. Dr. Shea receives grant support from NIH, the Department of Defense, and the Veterans Health Administration. Dr. Lyons has received consulting fees from GlaxoSmithKline, Novartis, Teva Neuroscience, Valeant, St. Jude Medical, Medtronic, and UCB/Schwarz Pharma. Dr. Pahwa has received honoraria or consulting fees from Boehringer Ingelheim, GlaxoSmithKline, Impax, Medtronic, Novartis, UCB, Teva Neuroscience, and Valeant. Dr. Adler has received grant support from Allergan, GlaxoSmithKline, Novartis, and has received consulting fees from Allergan, GlaxoSmithKline, Ipsen, and EMD Serono. Dr. Potenza participated in the following: consulted for and advised to Boehringer Ingelheim, received research support from Mohegan Sun and Forest Pharmaceuticals, had financial interests in Somaxon, and had consulted for law offices and the federal defender's office as an expert in pathological gambling and impulse control disorders. Dr. Hurtig received grant support from $\mathrm{NIH}$ and royalties from UpToDate. Dr. Siderowf has received consulting fees or honoraria from Boehringer Ingelheim, Merck Serono, and Teva. Dr. Duda has received honoraria from the Cooper University Health system and research grant support from the Department of Veterans Affairs, the National Institutes of Health, the Pennsylvania State Department of Health, Michael J. Fox Foundation for Parkinson Research and the Samueli Institute. Dr. Stern received consulting fees from Boehringer-Ingelheim, Novartis, NuPathe, Vernalis, Ipsen, Schering-Plough, and Teva.
} 
${ }^{5}$ Department of Medicine, University of Pennsylvania, Philadelphia, PA

${ }^{6}$ Center for Health Equity Research and Promotion (CHERP), Philadelphia Veterans Affairs Medical, Philadelphia, PA

7 Department of Neurology, University of Kansas Medical Center, Kansas City, KS

${ }^{8}$ Department of Neurology, Mayo Clinic Scottsdale, Scottsdale, AZ

${ }^{9}$ Department of Psychiatry, Yale University, New Haven, CT

${ }^{10}$ Division of Neurology, University of Toronto, Toronto, Canada

${ }^{11}$ National Institute of Neurological Disorders and Stroke (NINDS), Bethesda, MD

\section{Abstract}

Objective-As no comprehensive assessment instrument for impulse control disorders (ICDs) in Parkinson's disease (PD) exists, the aim of this study was to design and assess the psychometric properties of a self-administered screening questionnaire for ICDs and other compulsive behaviors in PD.

Methods-The Questionnaire for Impulsive-Compulsive Disorders in Parkinson's Disease (QUIP) has 3 sections: Section 1 assesses four ICDs (involving gambling, sexual, buying, and eating behaviors), Section 2 other compulsive behaviors (punding, hobbyism and walkabout), and Section 3 compulsive medication use. For validation, a convenience sample of 157 PD patients at 4 movement disorders centers first completed the QUIP, and then was administered a diagnostic interview by a trained rater blinded to the QUIP results. A shortened instrument (QUIP-S) was then explored.

Results-The discriminant validity of the QUIP was high for each disorder or behavior (receiver operating characteristic area under the curve [ROC AUC]: gambling $=0.95$, sexual behavior $=0.97$, buying $=0.87$, eating $=0.88$, punding $=0.78$, hobbyism $=0.93$, walkabout $=0.79$ ). On post hoc analysis, the QUIP-S ICD section had similar properties (ROC AUC: gambling $=0.95$, sexual behavior $=0.96$, buying $=0.87$, eating $=0.88$ ). When disorders/behaviors were combined, the sensitivity of the QUIP and QUIP-S to detect an individual with any disorder was $96 \%$ and $94 \%$, respectively.

Conclusions-Scores on the QUIP appear to be valid as a self-assessment screening instrument for a range of ICDs and other compulsive behaviors that occur in PD, and a shortened version may perform as well as the full version. A positive screen should be followed by a comprehensive, clinical interview to determine the range and severity of symptoms, as well as need for clinical management.

\section{Keywords}

Parkinson's disease; impulse control disorders; dopamine dysregulation syndrome; punding; pathological gambling

\section{INTRODUCTION}

Impulse control disorders (ICDs) are a group of psychiatric disorders whose essential feature is the failure to resist an impulse, drive, or temptation to perform an act harmful to either the self or others (1). ICDs that have been reported to occur in Parkinson's disease (PD) include compulsive gambling, buying, sexual, and eating behaviors(2).

Case reporting(3) and cross-sectional studies(4-6) suggest that prevalence estimates for ICDs may be higher in Parkinson's disease (PD) as compared with the general population(7-13) or with healthy control subjects(14;15), and it is not unusual for a patient to have multiple ICDs (16). Preliminary prevalence (either current or anytime during PD) estimates for ICDs in PD patients overall are $1.7-6.0 \%$ for problem or pathological gambling, $2.0-10.0 \%$ for compulsive 
sexual behavior, and $0.4-1.5 \%$ for compulsive buying $(2 ; 17 ; 18)$; there are no formal prevalence estimates for compulsive or binge-eating in PD. There is strong evidence of an association between dopamine agonist (DA) use and a range of ICDs and other compulsive behaviors in $\operatorname{PD}(2 ; 4 ; 6)$.

Other compulsive behaviors reported in PD include: (1) dopamine dysregulation syndrome (DDS) or hedonic homeostatic dysregulation, an addiction-like state marked by compulsive dopaminergic medication usage, particularly levodopa and short-acting dopamine agonists (e.g., subcutaneous apomorphine)(19); (2) punding, an intense fascination with meaningless movements or activities (e.g., cleaning, examining objects, or arranging) reported in 1.4-13.8\% of PD patients(20;21); (3) hobbyism, a complex form of punding characterized by intense fascination with a specific activity or hobby (e.g. writing, repairing or dismantling things, working on projects, or computer use)(2); and (4) walkabout, defined as excessive, aimless wandering (walking or driving)(19).

As patients may not report such behaviors to their treating neurologist either due to embarrassment or because they do not suspect an association with PD pharmacotherapy, ICD behaviors appear to be under-recognized in clinical practice(4), potentially prolonging the psychosocial consequences associated with these disorders. Thus, an instrument to screen for the range of ICDs and other compulsive behaviors is necessary to ensure their detection. However, no such instrument has been developed and validated for use in PD. The Questionnaire for Impulsive-Compulsive Disorders in Parkinson's Disease (QUIP) was designed with the goal of having a brief, self-completed screening instrument for use in clinical care and clinical research that covers the range of impulsive-compulsive behaviors reported in PD.

\section{METHODS}

\section{Questionnaire development}

First, existing screening and diagnostic instruments for ICDs and other compulsive behaviors that have been used in PD and the general population were reviewed(1;6;19;21-23). Second, input was solicited from outside experts in the area of ICDs in PD (MNP, JM, and VV) and from an expert in questionnaire development (JAS). Third, a preliminary ICD section of the QUIP was structured to be consistent with diagnostic criteria or defining clinical characteristics as described in the Diagnostic and Statistical Manual of Mental Disorders (DSM-IV-TR)(1). This consisted of an introductory question and four additional questions that addressed cognitive symptoms, affective symptoms, lack of ability to reduce or stop the behaviors, and activities that enable continuation of the behaviors. The compulsive medication use section was modeled on both Giovannoni's proposed criteria for hedonistic homeostatic dysregulation and DSM-IV substance dependence criteria. While minor wording changes were made in subsequent drafts, the structure of these sections remained consistent throughout the instrument development process. The other compulsive behaviors section was designed with conciseness in mind (an introductory question for each of the three behaviors plus two common additional questions). Guiding principles in the design of the QUIP included making it self-administered, brief yet comprehensive, and consistent in wording across different ICDs and other compulsive behaviors.

Next, the preliminary QUIP was administered to a sample of healthy controls (10 research staff members who work with neurodegenerative disease and psychiatric populations), and modifications were made based on the feedback received. Finally, the QUIP was administered to five PD patients and their informed others, and additional modifications were made based on the feedback received from them. 
The final version that was validated queried about behaviors that occurred at any time since the onset of PD (either inactive or active) that lasted at least four weeks. We chose the time frame of "anytime during PD" due to the observation that a substantial number of PD patients who have experienced an ICD during PD are currently asymptomatic due to clinical management, but may be at elevated risk of developing an ICD in the future. Another version of the QUIP that queries only about active behaviors is also available; it is identical to the validated version except for the time frame queried. The final version of the QUIP is divided into three sections: (1) five questions (including an introductory question that defines and gives examples of problem behaviors) for the four ICDs reported in PD; (2) three distinct introductory questions and two common additional questions for hobbyism, punding, and walkabout; and (3) five questions (including an introductory question) for compulsive medication use. The Flesch-Kincaid Readability Test assessed the QUIP to require a $12^{\text {th }}$ grade reading level.

\section{Subjects for validation process}

A convenience sample of 157 patients with idiopathic PD was assessed at four movement disorders centers between December 2007 and April 2008 (see Table 1 for demographic and clinical characteristics). The diagnosis of PD was confirmed by the patient's movement disorder neurologist. Patients were identified either in the context of routine clinical care or on the basis of having an ICD sometime during PD; the latter was done to enrich the sample with ICD patients. The Institutional Review Board at each participating institution approved the study, and written informed consent was obtained from subjects prior to study participation.

\section{Validation process}

The QUIP instructs patients to answer questions based on behaviors lasting at least four weeks occurring anytime after PD onset. After completing the QUIP, the patient was administered a "gold standard", semi-structured, diagnostic interview for compulsive gambling(1), buying (23), sexual behavior(6), eating(1), DDS(19), punding(19), hobbyism(2), and walkabout(19). Compulsive gambling included those patients with either problem or pathological gambling based on recommended cut-off points(24). The DSM-IV-TR research criteria for binge-eating disorder were modified to include general overeating in addition to discrete binge-eating episodes. The original criteria for walkabout were modified to exclude akathisia in order to capture purposeless wandering rather than a physical sensation of restlessness.

Prior to study initiation, each site identified research staff to administer the diagnostic interview who would be blinded to the results of the ICD questionnaire and unaware of the patient's ICD history. In addition, all raters administering the diagnostic interview received in-person or telephone training from the primary investigator (DW) on how to apply the diagnostic criteria for each disorder or behavior.

\section{Analyses}

A receiver operating characteristic (ROC) curve was plotted for each of the disorders/ behaviors. The area under curve (AUC), sensitivity, specificity, positive predictive value (PPV), and negative predictive value (NPV) were calculated for each disorder/behavior. The optimal cutoff score for each disorder/behavior was the point of maximum combined sensitivity and specificity; in order for a screening instrument to effectively differentiate individuals with and without an ICD, it should have high sensitivity and specificity (i.e., discriminant validity), as this maximizes the proportion of patients whose test results are accurate. Post hoc analyses were conducted to determine if a shortened version of the QUIP (QUIP-S) had similar psychometric properties to the full questionnaire. 


\section{RESULTS \\ ICD frequencies}

Frequencies of the different disorders and behaviors sometime during PD based on the diagnostic interview were: problem or pathological gambling (7.0\%), compulsive sexual behavior (8.9\%), compulsive buying (6.4\%), compulsive eating (4.5\%), punding $(10.2 \%)$, hobbyism (14.6\%), walkabout (3.2\%), and compulsive medication use $(<1.0 \%)$.

Overall, $31.2 \%$ of patients had a history of one or more ICDs, other compulsive behaviors, or compulsive medication use sometime during the course of PD. Approximately half of those subjects (15.9\% of entire sample) had a history of a single disorder or behavior, and the other half $(15.3 \%)$ had a history of two or more disorders or behaviors. Punding and hobbyism were usually distinct behaviors, with $18.5 \%$ of the population having only punding $(7.0 \%)$ or hobbyism (11.5\%), and $3.2 \%$ of the population having both punding and hobbyism.

The median completion time for the QUIP was 5 minutes.

\section{QUIP}

ICD section-The optimal cutoff point (i.e., point of maximum combined sensitivity and specificity) for each ICD was: (1) gambling: affirmative answers to $\geq 2$ questions; (2) sexual behavior: $\geq 1$ questions; (3) buying: $\geq 1$ questions; and (4) eating: $\geq 2$ questions (Table 2). These cut-off points provided at least $80 \%$ sensitivity and specificity for each ICD.

Other compulsive behaviors sections-For other compulsive behaviors, each introductory question by itself provided optimal sensitivity and specificity (Table 3). The discriminant validity of the single question for hobbyism was similar to that for the ICD section (sensitivity $=0.96$, specificity $=0.90$ ), while the questions for punding and walkabout had similarly high specificity but lower sensitivity.

Affirmative answers to the compulsive medication use questions were uncommon, ranging from $0-6.4 \%$. The sole patient who met criteria for compulsive medication use endorsed both the introductory question and the most commonly-endorsed of the other four questions.

Combining disorders/behaviors-As some patients were diagnosed with more than one ICD but did not endorse questions on the QUIP for all diagnosed ICDs, we assessed the validity of a positive response for any ICD (using the aforementioned cutoff points) to identify an individual with any $\geq 1 \mathrm{ICD}$ (as opposed to examining each individual ICD). This analysis had AUC $=0.88$ (sensitivity $=0.97$, specificity $=0.79, \mathrm{PPV}=0.53$, and $\mathrm{NPV}=0.99$ ).

Similarly, as some patients were diagnosed with more than one ICD or other compulsive behavior but did not endorse questions on the QUIP for each diagnosed disorder or behavior, we assessed the validity of a positive response for any ICD or other compulsive behavior (using the aforementioned cutoff points) to identify an individual with any $\geq 1$ ICD or other compulsive behavior (compulsive medication use excepted). This analysis had AUC $=0.85$ (sensitivity $=0.96$, specificity $=0.73, \mathrm{PPV}=0.62$, and $\mathrm{NPV}=0.98$ ).

QUIP-S

Item Selection-To evaluate a shortened version of the ICD section, we started with the introductory question for each ICD and added questions only if they increased the sensitivity of the instrument. As a result, the abbreviated ICD section has 2 questions for each disorder ( 8 total questions; Table 4 ). The optimal cutoff point for each ICD was $\geq 1$ affirmative answer 
to any question, which led to similar AUCs, sensitivities, and specificities as the full ICD section.

The complete QUIP-S, created based on the results mentioned above, consists of two questions for each of the four ICDs, the three introductory questions for other compulsive behaviors, and the two questions for compulsive medication use endorsed by the sole patient who met diagnostic criteria (for a total of 13 questions). A single positive response to any disorder's/ behavior's question is a positive screen for that disorder or behavior.

Combining disorders/behaviors-As we did for the QUIP, we assessed the validity of a positive response for any ICD (using the aforementioned optimal cutoff points of $\geq 1$ affirmative answer) in the QUIP-S ICD section to identify an individual with any $\geq 1$ ICD (as opposed to examining each individual ICD). This analysis had AUC=0.89 (sensitivity=1.00, specificity $=0.79, \mathrm{PPV}=0.53$, and $\mathrm{NPV}=1.00$ ).

We also assessed the validity of a positive response for any ICD or other compulsive behavior on the QUIP-S to identify an individual with any $\geq 1$ ICD or other compulsive behavior (with the exception of compulsive medication). This analysis had $\mathrm{AUC}=0.83$ (sensitivity $=0.94$, specificity $=0.72, \mathrm{PPV}=0.61$, and $\mathrm{NPV}=0.96$ ).

\section{DISCUSSION}

We found that the QUIP, a brief, self-completed screening questionnaire for ICDs and other compulsive behaviors in PD, has good discriminant validity using formal diagnostic criteria as the "gold standard" diagnosis.

To our knowledge, this is the first instrument to be developed and have score performance supporting validity as a screening instrument for the range of ICDs and other compulsive behaviors reported in PD. The Minnesota Impulsive Disorders Interview (MIDI)(22), which includes sections for compulsive gambling, sexual behavior, and buying, has been used in PD $(4 ; 17)$. However, the MIDI does not cover compulsive eating or other compulsive behaviors, and thresholding of scores to identify cases has varied across studies. Different rating scales (e.g., the South Oaks Gambling Screen(25)), questionnaires (Punding Questionnaire(20)), and diagnostic criteria (DSM-IV diagnostic criteria for pathological gambling, McElroy criteria for compulsive buying(26), Voon criteria for compulsive sexual behavior)(6), and descriptions of other compulsive behaviors $(19 ; 27)$ have either been used( $3 ; 5 ; 17 ; 28-30)$ or created for use $(6 ; 19 ; 20 ; 27)$ in PD, but no existing single instrument fulfills the criteria of being comprehensive, self-rated, and validated for use in this population.

The ICD section of the QUIP had at least $80 \%$ sensitivity and specificity for each of the 4 ICDs at the recommended cut-off points. As ICDs were frequently co-morbid but not always coendorsed, combining the four ICDs increased the sensitivity for identifying an individual with any ICD to $97 \%$. Thus, in many cases, ICD patients who failed to screen positive for one ICD were still identified with a positive screen for another ICD.

The sensitivity and specificity for the hobbyism subsection were both $>90 \%$. The instrument was limited in sensitivity for the punding and walkabout subsections (60-65\%). However, interpretation of the walkabout results was limited by the low number of cases. Furthermore, the full meaning and range of behaviors associated with punding were difficult to convey and capture in a brief questionnaire. Combining the ICD section and the other compulsive behaviors sections increased the sensitivity for identifying an individual with any disorder/behavior to $96 \%$. 
On post hoc analysis we found that the psychometric properties for each ICD in the QUIP-S were similar to their counterparts in the QUIP. Additionally, the QUIP-S ICD section overall had a sensitivity of $100 \%$ for identifying a patient with at least one ICD, and the total QUIPS had a sensitivity of $94 \%$ for identifying a patient with at least one ICD or other compulsive behavior.

The median completion time for the QUIP (30 questions total) was 5 minutes. We estimate that the median completion time for the QUIP-S (13 questions total) is 3 minutes. Although the shortened version was not formally tested, we do recommend it for routine use as the specific questions and overall structure of the instrument were not modified in any way. Clinicians or clinical researchers who want the additional information provided by the full questionnaire may choose to administer this version.

The QUIP was designed and validated as a screening instrument, not as a diagnostic or rating instrument. The negative predictive values (NPVs) for each ICD were very high, so a negative screen appears to signal with a great degree of certainty that an ICD is not present. For a screening instrument, a high NPV is crucial, while a low PPV can be counterbalanced by conducting a follow-up clinical interview.

Positive predictive values (PPVs) were low overall, indicating that a positive screen needs to be followed by a clinical interview to verify if the patient truly has clinically significant ICD or other compulsive behaviors. Since ICDs and other compulsive behaviors are commonly comorbid, patients in particular who screen positive for a single ICD should be queried about the range of ICDs and other compulsive behaviors reported in PD.

The low PPVs for most of the ICDs and other compulsive behaviors are in part a reflection of the relatively low frequency of each disorder in our study population (the most common behavior, hobbyism, was diagnosed in $<15 \%$ of patients). In addition, there are other reasons a patient might endorse symptoms on the QUIP but not meet diagnostic criteria for an ICD or other compulsive behavior when interviewed. First, ICDs or other compulsive behaviors may be present at subsyndromal levels, in which case follow-up and monitoring is appropriate as such patients may be at higher risk of developing a disorder (i.e., having symptoms that lead to distress or some form of psychosocial impairment). Similarly, a clinician might consider the presence of subsyndromal symptoms in therapeutic decisions for a given patient, such as whether to utilize a DA or levodopa. Second, a patient may acknowledge symptoms leading to a positive screen with the QUIP, but minimize symptom severity on formal interview leading to a negative diagnostic interview. An example from clinical experience are married male patients who acknowledge compulsive sexual behaviors, but report them as not being clinically significant, whereas the spouse will report that such symptoms are causing distress or impairment in the marital relationship. This situation highlights the value of including an informed other, if available, when clinically evaluating the significance of ICD and other compulsive behaviors. Third, the diagnostic criteria used for the different disorders for the validation process may have imposed a limitation. Pathological gambling has arguably the most well-established diagnostic criteria(1), and the QUIP had the highest PPV for this disorder. The lowest PPVs were for compulsive buying and eating, disorders for which it may be difficult to differentiate pathological behaviors from excessive behaviors that do not rise to the level of a disorder. Finally, a patient may be more willing to endorse symptoms on a selfadministered questionnaire than to an interviewer.

There are several limitations to note. First, the diagnostic criteria for two of the disorders and behaviors (i.e., binge-eating disorder and walkabout) were slightly modified to be consistent with clinical experience. Second, only one patient met diagnostic criteria for compulsive medication use thus limiting validation of this section of the questionnaire, and less than 10 
subjects each had compulsive eating or walkabout. Third, as we used a time frame of "anytime during PD" to assess ICD and other compulsive behaviors, recall bias may have influenced the accuracy of the information provided. Fourth, as our study population was a convenience sample of PD patients, we cannot say if the instrument would have performed differently in a random sample of patients. Finally, as a result of providing many examples and using terms from existing instruments, the QUIP is rated at a $12^{\text {th }}$ grade reading level, and it is unknown how this would affect the screening of PD patients with less than 12 years of formal education.

Both the QUIP and QUIP-S are appropriate for use in clinical care and clinical research as screening instruments for the range of ICDs and other compulsive behaviors reported in PD. Future studies should include a larger number of patients with a range of ICD histories to validate our preliminary findings. In addition, the QUIP should be tested in other populations who may be at risk for ICD development (e.g., restless leg syndrome [RLS] patients receiving DA therapy(31)).

\section{Supplementary Material}

Refer to Web version on PubMed Central for supplementary material.

\section{Acknowledgments}

Sarra Nazem and Eugenia Mamikonyan (University of Pennsylvania) for their assistance in the design of the QUIP, subject recruitment and data collection; April Langhammer (University of Kansas Medical Center) for her assistance in subject recruitment and data collection; and Bryce Falk and Charlene Hoffman-Snyder (Mayo Clinic Scottsdale) for their assistance in subject recruitment and data collection.

\section{References}

1. American Psychiatric Association. Diagnostic and Statistical Manual of Mental Disorders. 4. Washington, DC: American Psychiatric Association; 2000. Text Revision

2. Voon V, Fox SH. Medication-related impulse control and repetitive behaviors in Parkinson disease. Arch Neurol 2007;64:1089-96. [PubMed: 17698698]

3. Dodd ML, Klos KJ, Bower JH, et al. Pathological gambling caused by drugs used to treat Parkinson disease. Arch Neurol 2005;62:1-5.

4. Weintraub D, Siderowf AD, Potenza MN, et al. Association of dopamine agonist use with impulse control disorders in Parkinson disease. Arch Neurol 2006;63:969-73. [PubMed: 16831966]

5. Voon V, Hassan K, Zurowski M, et al. Prospective prevalence of pathological gambling and medication association in Parkinson disease. Neurology 2006;66:1750-1752. [PubMed: 16769956]

6. Voon V, Hassan K, Zurowski M, et al. Prevalence of repetitive and reward-seeking behaviors in Parkinson disease. Neurology 2006;67:1254-57. [PubMed: 16957130]

7. Pietrzak RH, Morasco BJ, Blanco C, Grant BF, Petry NM. Gambling level and psychiatric and medical disorders in older adults: results from the National Epidemiologic Survey on Alcohol and Related Conditions. Am J Geriatr Psychiatry 2007;15:301-13. [PubMed: 17095749]

8. Stucki S, Rihs-Middel M. Prevalence of adult problem and pathological gambling between 2000 and 2005: an update. Journal of Gambling Studies 2008;23:245-57.

9. Volberg, RA.; Nysse-Carris, KL.; Gerstein, DR. 2006 California Problem Gambling Prevalence Survey. National Opinion Research Center at the University of Chicago; 2006.

10. Shaffer HJ, Hall MN, Vander J. Estimating the prevalence of disordered gambling behavior in the United States and Canada: a research synthesis. Am J Public Health 1999;89:1369-76. [PubMed: 10474555]

11. Black DW. A review of compulsive buying disorder. World Psychiatry 2007;6:14-18. [PubMed: 17342214]

12. Lilenfeld LRR, Ringham R, Kalarchian MA, Marcus MD. A family history study of binge-eating disorder. Compr Psychiatry 2008;49:247-54. [PubMed: 18396183] 
13. Coleman E. Is your patient suffering from compulsive sexual behavior? Psychiatric Annals 1992;22:320-325.

14. Giladi N, Weitzman N, Schreiber S, Shabtai H, Peretz C. New onset heightened interest or drive for gambling, shopping, eating or sexual activity in patients with Parkinson's disease: the role of dopamine agonist treatment and age at motor symptoms onset. J Psychopharmacol (Oxf) 2007;21:501-6.

15. Avanzi M, Baratti M, Cabrini S, Uber E, Brighetti G, Bonfà F. Prevalence of pathological gambling in patients with Parkinson's disease. Mov Disord 2006;21:2068-72. [PubMed: 17044068]

16. Singh A, Kandimala G, Dewey RB, O’Suilleabhain P. Risk factors for pathological gambling and other compulsions among Parkinson's disease patients taking dopamine agonists. Journal of Clinical Neuroscience 2007;14:1178-81. [PubMed: 17720504]

17. Isaias IU, Siri C, Cilia R, De Gaspari D, Pezzoli G, Antonini A. The relationship between impulsivity and impulse control disorders in Parkinson's disease. Mov Disord 2008;23:411-15. [PubMed: 18067187]

18. Pontone G, Williams JR, Bassett SS, Marsh L. Clinical features associated with impulse control disorders in Parkinson disease. Neurology 2006;67:1258-61. [PubMed: 17030761]

19. Giovannoni G, O’Sullivan JD, Turner K, Manson AJ, Lees AJL. Hedonistic homeostatic dysregulation in patients with Parkinson's disease on dopamine replacement therapies. J Neurol Neurosurg Psychiatry 2000;68:423-28. [PubMed: 10727476]

20. Evans AH, Katzenschlager R, Paviour D, et al. Punding in Parkinson's disease: its relation to the dopamine dysregulation syndrome. Mov Disord 2004;19:397-405. [PubMed: 15077237]

21. Miyasaki J, Hassan KL, Lang AE, Voon V. Punding prevalence in Parkinson's disease. Mov Disord 2007;22:1179-81. [PubMed: 17230464]

22. Christenson GA, Faber RJ, deZwaan M. Compulsive buying: descriptive characteristics and psychiatric comorbidity. J Clin Psychiatry 1994;55:5-11. [PubMed: 8294395]

23. Lejoyeux M, Tassain V, Solomon J, Adès J. Study of compulsive buying in depressed patients. J Clin Psychiatry 1997;58:169-73. [PubMed: 9164428]

24. Fisher SE. Gambling and Problem Gambling Among Casino Patrons. Report to the British Casino Industry Consortium. 1996

25. Lesieur HR, Blume SB. The South Oaks Gambling Screen (SOGS): a new Instrument for the identification of pathological gamblers. Am J Psychiatry 1987;144:1184-88. [PubMed: 3631315]

26. McElroy SL, Keck PE, Pope HG, Smith JM, Strakowski SM. Compulsive buying: a report of 20 cases. J Clin Psychiatry 1994;55:242-48. [PubMed: 8071278]

27. Pezzella FR, Di Rezze S, Chianese M, et al. Hedonistic homeostatic dysregulation in Parkinson's disease: a short screening questionnaire. Neurological Sciences 2003;24:205-6. [PubMed: 14598089]

28. Voon V, Thomsen T, Miyasaki JM, et al. Factors associated with dopaminergic drug-related pathological gambling in Parkinson disease. Arch Neurol 2007;64:212-16. [PubMed: 17296836]

29. Grosset KA, Macphee G, Pal G, et al. Problematic gambling on dopamine agonists: not such a rarity. Mov Disord 2006;21:2206-8. [PubMed: 17013907]

30. Klos KJ, Bower JH, Josephs KA, Matsumoto JY, Ahlskog JE. Pathological hypersexuality predominantly linked to adjuvant dopamine agonist therapy in Parkinson's disease and multiple system atrophy. Parkinsonism and Related Disorders 2005;11:381-86. [PubMed: 16109498]

31. Ondo W, Lai D. Predictors of impulsivity and reward seeking behavior with dopamine agonists. Parkinsonism and Related Disorders 2008;14:28-32. [PubMed: 17702628] 


\section{Table 1}

Demographic and clinical characteristics

\begin{tabular}{|l|l|}
\hline Variable & Mean (SD) or \% (N) \\
\hline Age (\# years) & $65(9.4)$ \\
\hline Sex (\% male) & $75 \%(118)$ \\
\hline Race (\% white) & $95 \%(149)$ \\
\hline Education (\# years) & $15.4(3.0)$ \\
\hline PD duration (\# years) & $9.0(6.3)$ \\
\hline Hoehn \& Yahr stage (median) & 2.0 \\
\hline Levodopa dosage (mg/day) & $618(434)$ \\
\hline Dopamine agonist use (\% yes) & $59 \%(93)$ \\
\hline History of deep brain stimulation (DBS; \% yes) & $20 \%(32)$ \\
\hline Self-report history of bipolar disorder (\% yes) & 0 \\
\hline
\end{tabular}




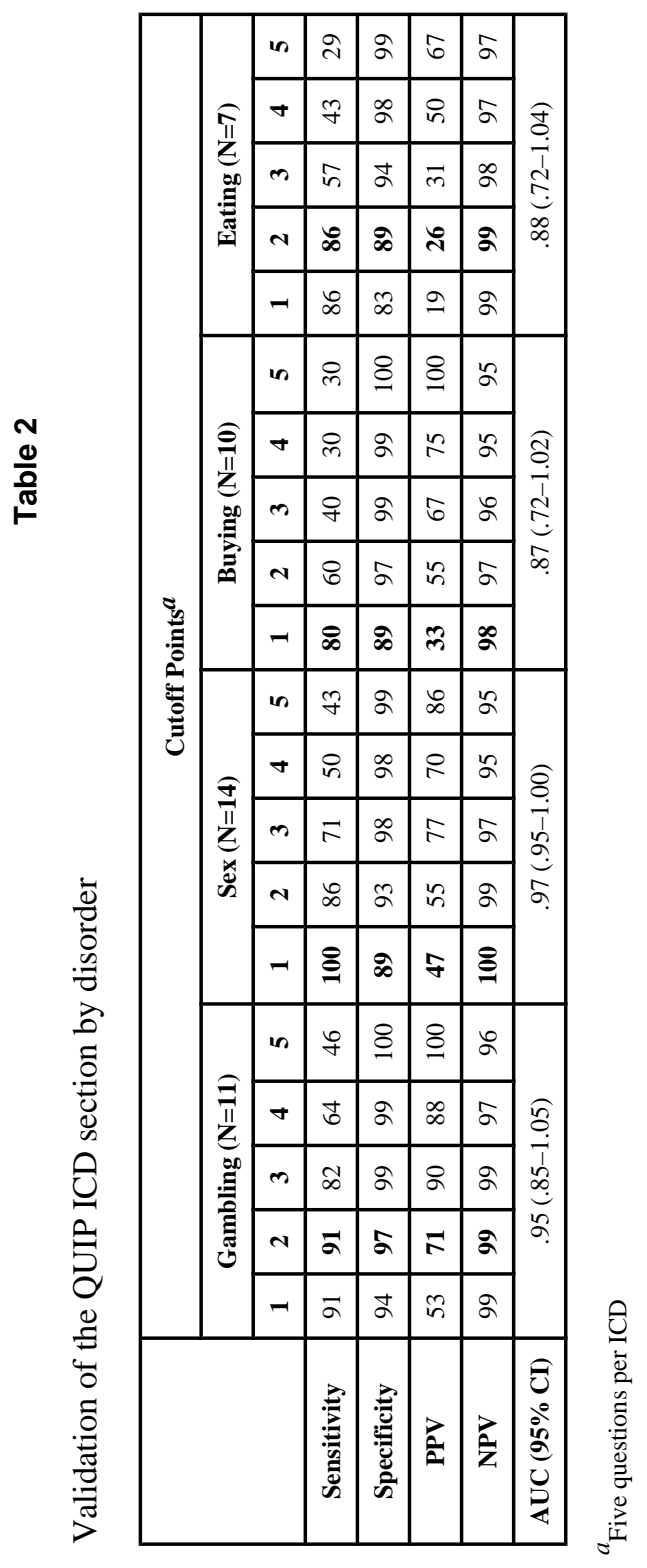

Mov Disord. Author manuscript; available in PMC 2010 April 4. 
Table 3

Validation of other compulsive behaviors

\begin{tabular}{|c|c|c|c|}
\hline \multirow{2}{*}{} & \multicolumn{3}{|c|}{ Introductory Questions } \\
\cline { 2 - 4 } & Hobbyism (N=23) & Punding (N=16) & Walkabout (N=5) \\
\hline Sensitivity & 96 & 63 & 60 \\
\hline Specificity & 90 & 93 & 97 \\
\hline PPV & 61 & 50 & 43 \\
\hline NPV & 99 & 96 & 99 \\
\hline AUC $(\mathbf{9 5 \%}$ CI) & $.93(.87-.98)$ & $.78(.63-.92)$ & $.79(.52-1.05)$ \\
\hline
\end{tabular}




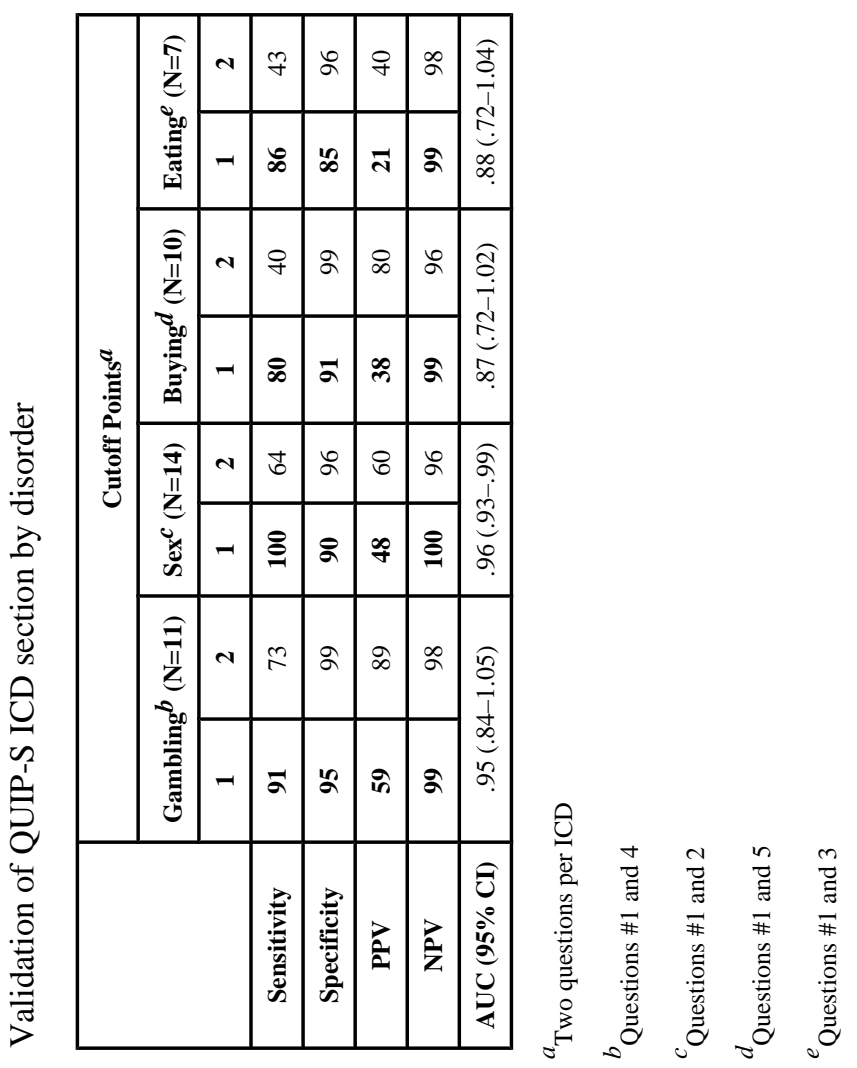

Mov Disord. Author manuscript; available in PMC 2010 April 4. 